\title{
DC Proposal: PRISSMA, Towards Mobile Adaptive Presentation of the Web of Data
}

\author{
Luca Costabello \\ INRIA Edelweiss Team, Sophia Antipolis, France \\ luca.costabello@inria.fr
}

\begin{abstract}
The Mobile Web is evolving fast and mobile access to the Web of Data is gaining momentum. Interlinked RDF resources consumed from portable devices need proper adaptation to the context in which the action is performed. This paper introduces PRISSMA (Presentation of Resources for Interoperable Semantic and Shareable Mobile Adaptability), a domain-independent vocabulary for displaying Web of Data resources in mobile environments. The vocabulary is the first step towards a declarative framework aimed at sharing and re-using presentation information for context-adaptable user interfaces over RDF data.
\end{abstract}

Keywords: Mobile Web of Data, RDF Presentation, Adaptive UI.

\section{Introduction}

The Mobile Web and the Web of Data are strong trends for the next evolution of the web. Ubiquitous access is growing fast, as a result of heterogeneous factors such as new generation access networks, increasing device capabilities, novel interaction paradigms and enhanced user interfaces 1 . At the same time, the Linked Data Initiative 2 contributes to the growing popularity of the world of interlinked, structured and open data on the web. Soon mobile applications will offer novel ways of consuming and contributing to the Web of Data, both with the adoption of novel interaction modalities (e.g. enhanced Mobile Web applications, voice interaction with the web, augmented reality, etc.) and, on the other hand, with a deeper awareness of the surrounding physical environment.

Mobile Web of Data consumption faces the same general issues of classic Webof-Data-savvy applications, such as access strategy choice, vocabulary mapping, identity resolution, provenance and data quality assessment 10. Another wellknown problem affecting Web of Data applications consists in delivering effective user interfaces for RDF resources. When dealing with mobile devices, the issue of adaptation arises: classic Mobile Web experience suggests that the same information is not meant to be represented in the same way on all devices. Moreover, context-awareness research underlines the influence of the surrounding environment on data representation [481213]. The same principles and best practices

1 http://pewinternet.org/Reports/2011/Smartphones.aspx

2 http://linkeddata.org/

L. Aroyo et al. (Eds.): ISWC 2011, Part II, LNCS 7032, pp. 269 276, 2011.

(C) Springer-Verlag Berlin Heidelberg 2011 
apply to RDF resource representation in mobile environments, as shown in the following foaf : Person-related scenario.

Alice loves shopping. Today is at the shopping mall, looking for a birthday present for Bob. She needs an idea, so she opens Bob's FOAF profile looking for inspiration. Alice's smartphone is aware of being at the mall, and therefore displays Bob's interests while hiding other properties such as his email or geographical position. As she exits the mall and walks down the street, her smartphone visualizes Bob's profile as a foaf : Depiction on a map. In the meanwhile, Bob is jogging. The mobile in his pocket updates him on the status of his FOAF contacts using speech synthesis. Each contact's latest social status update is read out loud. Bob listens the audio reproduction of Alice's last tweet, and decides to reply. As he stops running and takes the device into hands, the smartphone deactivates the audio representation and adopts a visual paradigm listing Alice's phone number, email and social network accounts.

RDF consumption in mobile environments needs contextual resource adaptation and heterogeneous dimensions must be involved (e.g. the mobile user profile, the device features and the surrounding physical environment). Representation paradigm independence has to be provided, thus supporting multi-modality. Moreover, tools designed for RDF resource adaptation on mobile devices must not rely on ad-hoc solutions. Re-use must be favoured and encouraged, thus enabling the exchange of context-based knowledge across heterogeneous applications. This paper sets the basis for a Web of Data mobile presentation framework, and it focuses on the PRISSMA lightweight vocabulary3, an early stage contribution providing classes and properties to model core mobile context concepts useful for dynamic adaptation of user interfaces for RDF data.

The remainder of the paper is organized as follows: Section 2 provides a state of the art overview. Section 3 describes with further details the issue of mobile adaptive RDF representation and presents the research plan. Section 4 describes the PRISSMA vocabulary, an early contribution. In Section 5] discussion of the approach is provided, along with the most relevant perspectives.

\section{Related Work}

Related work includes proposals from heterogeneous research domains, such as RDF presentation, content adaptation in mobile classic web and context-aware data representation.

Fresnel [14 is a vocabulary modelling core presentation-level concepts for RDF visualization. The assumption is that data and its related schema do not carry sufficient information for representation: further presentation knowledge has to be provided. This information is expressed in RDF as well, in order to ensure homogeneity and favour presentation knowledge sharing among different applications. In Fresnel, Lens components select and filter information while Formats define how to present data. Designed for static environments, Fresnel leaves contextual adaptation of RDF data to the application logic. Sharing and

\footnotetext{
3 http://ns.inria.fr/prissma
} 
re-using contextual conditions for RDF presentation is therefore not possible. More recent proposals targeting RDF user interfaces have been provided, both of them being RDF template systems [12. In LESS, the authors propose an endto-end approach to consume linked data based on the Smarty template engine 1]. Dadzie et al. suggest a template-based RDF visualization approach aimed at facilitating Web of Data consumption 2. Although they move in the direction of easing the development of Web of Data applications, these approaches do not address the specific needs of mobile environments, e.g. context adaptability.

Initiatives such as the now discontinued W3C Model-Based User Interface Incubator Group deal with classic Mobile Web content adaptation (i.e. RDF consumption-related presentations issues are not considered) 6]. The declared goal is to 'evaluate research on model-based user interface design' for the authoring of web applications. The authors propose the Delivery Context Ontology, a modular, fine-grained vocabulary to model mobile platforms [7].

CAMB is a mobile context-aware HTML browser that adapts web pages according to a predetermined set of environmental situations 8]. Other proposals have been made in this direction, e.g. 41213. None of these address a Web-ofData-specific scenario.

The approach proposed in the remainder of the paper adapts and extends to the mobile world (static) Web of Data representation best practices, such as those described in Fresnel [14.

\section{Towards Mobile Adaptive RDF Representation}

Classic web content needs ad-hoc adaptation when displayed on portable devices and its visualization is influenced by the surrounding mobile context as well [8]. Web of Data access is no exception: whenever an RDF-fuelled mobile application needs to display some resources, adaptation (e.g., to the surrounding context) must be performed to deliver better user experience.

A widely-accepted formalization of context can be found in [3] and inspires the proposal in 6]. The authors of the latter describe the mobile context as an encompassing term, an information space defined as the sum of three different dimensions: the mobile User model, the Device features and the Environment in which the action is performed. This proposal is adopted as operative definition by the present work.

Dealing with Web of Data resources adaptation inside the application logic prevents the sharing of context-related presentation knowledge and intrinsically ties to a fixed representation paradigm. A common and shareable presentationlevel solution provides common ground for building mobile adaptive user interfaces, avoids re-inventing the wheel and favours the exchange of context-related presentation knowledge between mobile applications. The need for sharing and reuse contextual presentation is not compatible with hardwired, programmatic approaches: a declarative proposition must be adopted to favour presentation data reuse (the latter point being the main Fresnel objective [14]).

As mentioned in Section [1 mobile devices provide heterogeneous representation paradigms (not necessarily visual, e.g. voice). A presentation-level approach 
is independent from the representation paradigm chosen by the application, and it is therefore suitable for mobile applications that need to change their RDF representation paradigm dynamically (e.g. contextually switching from a nested boxes layout à la HTML to a timeline or voice output).

In terms of research plan, achieving contextual content adaptation on mobile devices includes the following steps:

Contextual Presentation-Level Model Definition. The overall approach relies on the PRISSMA vocabulary, used to specify in which context a given representation must be activated. PRISSMA is designed as an extension of Fresnel [14] and it is described as an early contribution in Section 4.

Comparison between Declared and Real Context. Every time a Web of Data resource is requested, PRISSMA-based applications need to compare declared activation contexts and real situations to select the proper visualization. Implementing a rendering solution based on PRISSMA introduces therefore a graph matching problem. On the front-end, this issue can be modeled as a SPARQL ASK query performed against a set of PRISSMA declarations, having the goal of looking for a match between the real, detected context and a declared item in the set (both real context and the declared ones included in the set are modelled with PRISSMA). The intrinsic nature of contextual data and PRISSMA lightweight approach determine the need for an approximate matching solution. In other words, the comparison must take into account the discrepancies between the declared context and the actual situation. The proposed strategy must rely on a compound notion of distance: heterogeneous dimensions need to be considered (e.g. location, time, terminological heterogeneity), therefore different metrics must be chosen, along with a proper composition function. The algorithm needs to be unsupervised and one-pass, in order to enable responsive mobile applications.

Specificity Computation Rules. Conflicts might occur in case more than one representation is suitable for the current situation: a specificity computation mechanism has to be provided. This could be implemented giving higher priority to the most detailed and specific PRISSMA declarations.

Presentation Knowledge Distribution. PRISSMA declarations can be published as linked data. A discovery mechanism is needed to enable mobile applications that rely on PRISSMA to potentially adopt representations shared on the web.

\section{PRISSMA Vocabulary Overview}

The PRISSMA vocabulary provides classes and properties to model core mobile context concepts useful for dynamic adaptation of user interfaces for RDF 


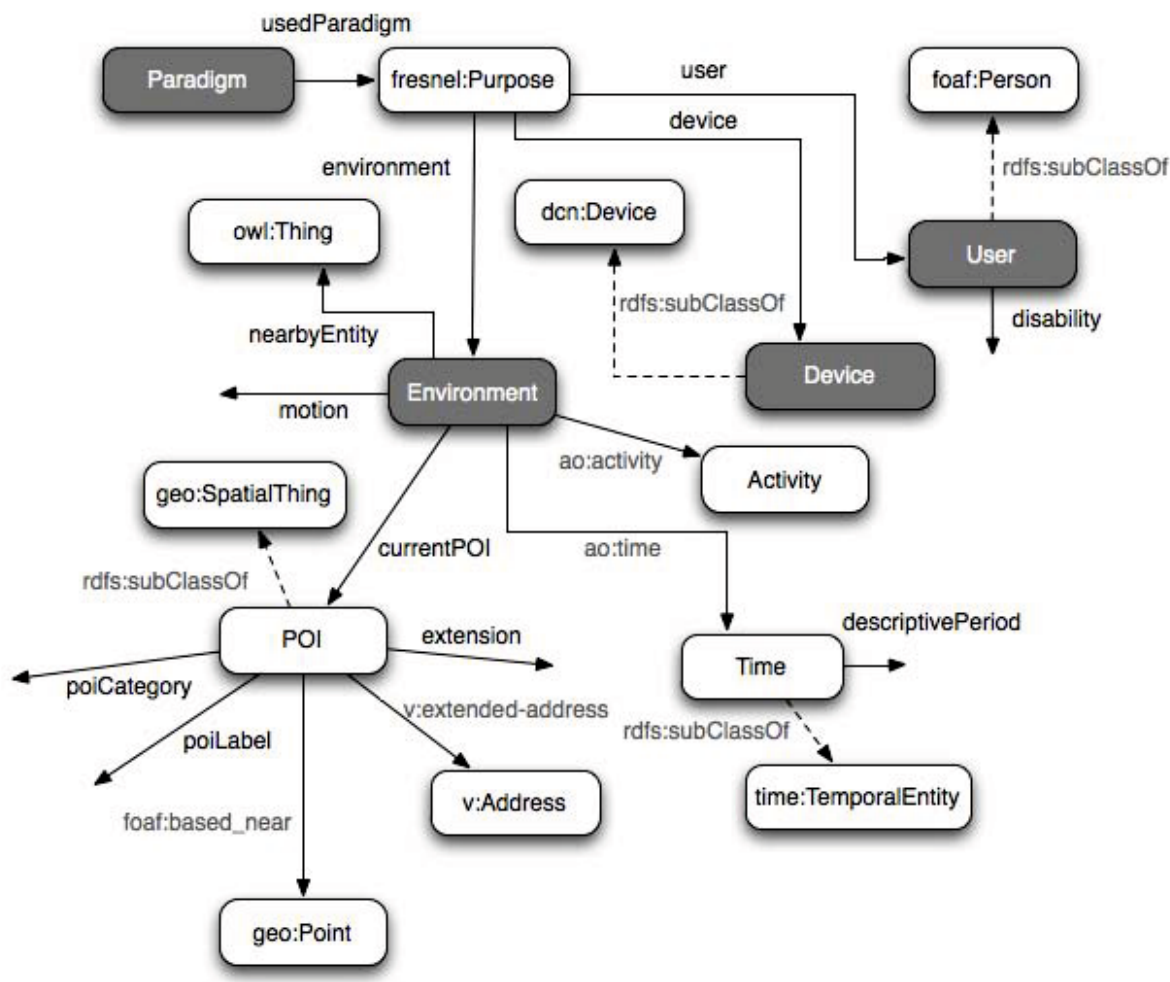

Fig. 1. The PRISSMA vocabulary at a glance. Grey boxes represent core classes.

data. Designed to work in conjunction with the Fresnel presentation vocabulary, PRISSMA specifies when (i.e. in which context) Fresnel Lenses and Formats must be activated. The original Fresnel core vocabulary includes the class fresnel: Purpose and the property fresnel:purpose, used to choose between lenses in conflicting situations, i.e. when more than one lens applies. Predefined instances of fresnel : Purpose are provided, e.g. defaultLens, labelLens. The extended Fresnel vocabulary provides instances such as screen, print and projection, in order to support a basic media-based presentation. PRISSMA re-uses fresnel : Purpose to model a contextual situation in which an associated Fresnel lens and format must be activated.

PRISSMA is based on the definition of context provided in Section 3 , it is not meant to provide yet another mobile contextual model, therefore well-known vocabularies are reused. Moreover, it does not provide an exhaustive set of properties for resource adaptation: (extensions are free to add further functionalities). Fresnel Lenses and Formats are showed according to the following dimensions:

${ }^{4}$ From latin prisma. In optics, a Fresnel lens can be considered as a series of prisms. 


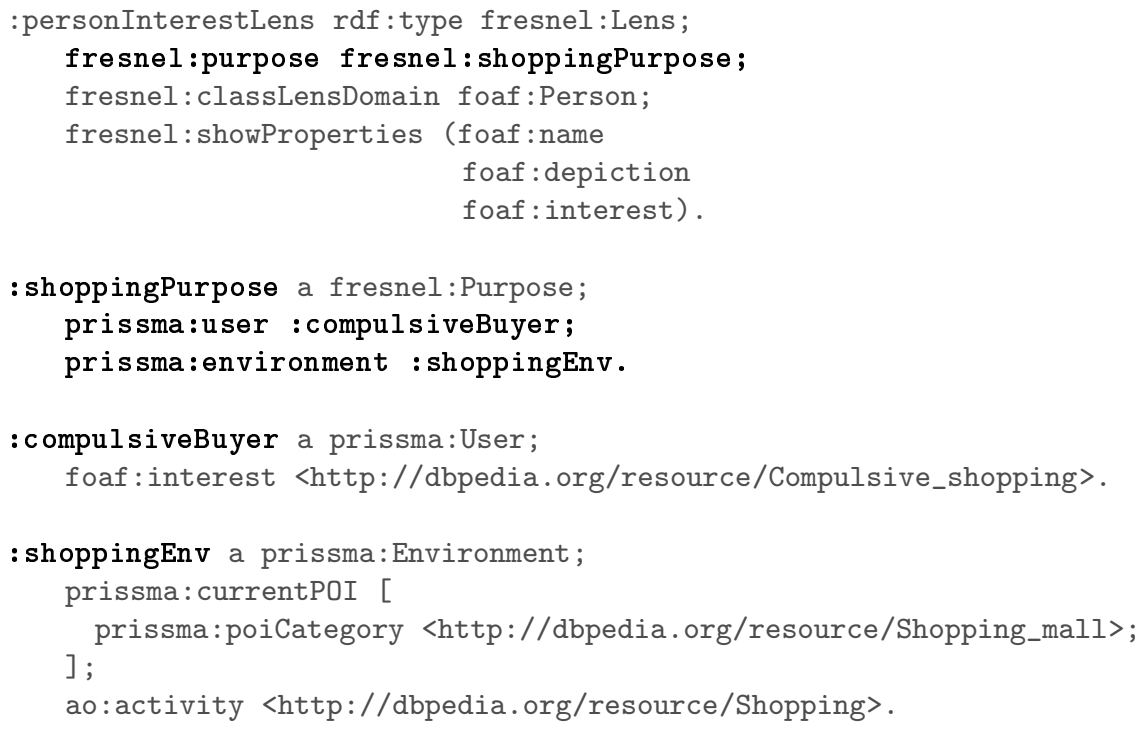

Fig. 2. A Fresnel Lens associated to a mobile context using PRISSMA

User. Represents the target mobile user associated to a fresnel:Purpose and consists in a foaf:Person sub-class. To provide more flexibility, the class can be used to model both user stereotypes and specific users.

Device. Represents the mobile device on which Web of Data resource consumption takes place, enabling device-specific data representation. The class inherits from W3C Delivery Context Ontology 7] dcn:Device that provides an extensible and fine-grained model for mobile device features.

Environment. Models the physical context in which the Web of Data resource consumption takes place, therefore enabling customized resource representation according to specific situations. Different dimensions are involved in modelling the surrounding environment. As anticipated in Section 3, PRISSMA does not provide a comprehensive, fine grained model. The approach is to delegate refinements and extensions to domain specialists.

Location is modelled with the notion of Point of Interest (POI). The prissma: POI class consists in a simplified, RDFized version of W3C Point of Interest Core specifications [15]. POIs are defined as entities that "describe information about locations such as name, category, unique identifier, or civic address". Each prissma:POI consists in a geo:SpatialThing and can be associated to a given geo: Point coupled with a physical radius via the prissma: extension property. The properties prissma:poiCategory and prissma: poiLabel are used to assign a category (e.g. http://dbpedia.org/resource/Shopping_mall) and a label (e.g. http://dbpedia.org/page/Harrods). Address details can be provided as well, using the vCard ontology class v:Address [9]. 
Temporal dimension is modelled extending the time:TemporalEntity class [11. The prissma: descriptivePeriod property associates a description to each Time entity (e.g. http://dbpedia.org/resource/Afternoon).

Other dimensions are considered: the motion property associates any given high-level representation of motion to an Environment. The environmental proximity of a generic object can trigger different resource representations: nearby objects are associated to the Environment with the prissma:nearbyEntity property. The prissma:Activity class consists in a placemark aimed at connecting third-party solutions focused on inferring high-level representations of user actions (e.g.'running', 'driving', 'working', 'shopping', etc.). The Association Ontology's ao:activity [5] is used to associate this class to the rest of the model.

Figure 2 provides an example related to the scenario presented in Section 1 personInterestLens is a Fresnel lens that shows the interests of a foaf :Person. The lens is activated by the purpose shoppingPurpose, which is defined by the prissma:User "compulsive buyer" and by the prissma:Environment "shopping in a mall".

The original Fresnel proposition does not specify the representation paradigm that the user agent should adopt (e.g. web page-based box model, voice output, etc). Fresnel implementations output a paradigm-independent abstract box model that is rendered appropriately by the application. Being able to address this aspect in a declarative way is important in a context-aware environment, where the representation paradigm might vary dynamically. The class prissma:Paradigm models therefore a Fresnel box model customized for a given representation paradigm. The property prissma:usedParadigm allows the association to a fresnel:Purpose.

\section{Discussion and Perspectives}

This paper presents an approach towards contextual adaptation of Web of Data resources on mobile devices and describes the PRISSMA vocabulary used as foundation.

Relying on a presentation-level approach guarantees independence from the chosen Web of Data access strategy: PRISSMA operates after this step, and is therefore compatible with the follow-your-nose approach, SPARQL endpoints querying and indexing services APIs. PRISSMA relies on Fresnel, and complies therefore to the well-known presentation vocabulary, thus adding context adaptability as a new scenario of use. A Fresnel-based approach favours the sharing and reuse of contextual presentation knowledge across mobile applications consuming the Web of Data. The adoption of RDF formalism guarantees homogeneity within presentation knowledge, i.e. context-related information are expressed in RDF and no other formalism is needed. An extensible vocabulary provides support to further contextual information.

On the other hand, it must not be underestimated that a context-savvy mobile presentation layer based on an RDF vocabulary introduces intrinsic complexity that has to be taken into account while developing context-adapting, RDF-based applications. 
The PRISSMA vocabulary and the algorithms solving the research steps described in Section 3 need to undergo a proper evaluation. A mobile Web of Data browser implementing the proposal described in this paper is to be provided as a test bench. The prototype is meant to contextually adapt the representation of requested instances, enabling therefore a testing campaign to compare the resulting adaptation of Web of Data resources to user expectations.

\section{References}

1. Auer, S., Doehring, R., Dietzold, S.: LESS - Template-Based Syndication and Presentation of Linked Data. In: Aroyo, L., Antoniou, G., Hyvönen, E., ten Teije, A., Stuckenschmidt, H., Cabral, L., Tudorache, T. (eds.) ESWC 2010. LNCS, vol. 6089, pp. 211-224. Springer, Heidelberg (2010)

2. Dadzie, A.-S., Rowe, M., Petrelli, D.: Hide the Stack: Toward Usable Linked Data. In: Antoniou, G., Grobelnik, M., Simperl, E., Parsia, B., Plexousakis, D., De Leenheer, P., Pan, J. (eds.) ESWC 2011, Part I. LNCS, vol. 6643, pp. 93-107. Springer, Heidelberg (2011)

3. Dey, A.K.: Understanding and using context. Personal Ubiquitous Computing 5, 4-7 (2001)

4. Eissele, M., Weiskopf, D., Ertl, T.: Interactive context-aware visualization for mobile devices. In: Butz, A., Fisher, B., Christie, M., Krüger, A., Olivier, P., Therón, R. (eds.) SG 2009. LNCS, vol. 5531, pp. 167-178. Springer, Heidelberg (2009)

5. Ferris, B., Inkster, T.: The Association Ontology 0.4 (2010), http://purl.org/ontology/ao/core\#

6. Fonseca, J.M.C., González Calleros, J.M., Meixner, G., Paternò, F., Pullmann, J., Raggett, D., Schwabe, D., Vanderdonckt, J.: Model-Based UI XG Final Report (2010), http://www.w3.org/2005/Incubator/model-based-ui/XGR-mbui/

7. Fonseca, J.M.C., Lewis, R.: Delivery Context Ontology, W3C Working Draft (2009), http://www.w3.org/TR/2009/WD-dcontology-20090616/

8. Gasimov, A., Magagna, F., Sutanto, J.: CAMB: context-aware mobile browser. In: Proceedings of the 9th International Conference on Mobile and Ubiquitous Multimedia, pp. 22:1-22:5. ACM (2010)

9. Halpin, H., Suda, B., Walsh, N.: An Ontology for vCards (2006), http://www.w3.org/2006/vcard/ns-2006.html

10. Heath, T., Bizer, C.: Linked Data: Evolving the Web into a Global Data Space, 1st edn. Morgan \& Claypool (2011)

11. Hobbs, J.R., Pan, F.: Time Ontology in OWL (2006), http://www.w3.org/TR/owl-time/

12. Lemlouma, T., Layaida, N.: Context-Aware Adaptation for Mobile Devices. In: IEEE International Conference on Mobile Data Management (2004)

13. Nathanail, S., Tsetsos, V., Hadjiefthymiades, S.: Sensor-Driven Adaptation of Web Document Presentation. In: Stephanidis, C. (ed.) HCI 2007. LNCS, vol. 4556, pp. 406-415. Springer, Heidelberg (2007)

14. Pietriga, E., Bizer, C., Karger, D., Lee, R.: Fresnel: A Browser-Independent Presentation Vocabulary for RDF. In: Cruz, I., Decker, S., Allemang, D., Preist, C., Schwabe, D., Mika, P., Uschold, M., Aroyo, L.M. (eds.) ISWC 2006. LNCS, vol. 4273, pp. 158-171. Springer, Heidelberg (2006)

15. Womer, M.: Points of Interest Core, W3C Working Draft (2011), http://www.w3.org/TR/poi-core/ 